\title{
Opiniões de mães de crianças que morreram no primeiro ano de vida no Município de Londrina, Paraná, Brasil
}

\author{
Opinions of mothers whose children died during \\ their first year of life in Londrina, Paraná, Brazil
}

Ana Maria Rigo Silva 1

Selma Maffei de Andrade 1

Zuleika Thomson 1

\footnotetext{
1 Departamento

Materno-Infantil e Saúde

Comunitária, Centro

de Ciências da Saúde,

Universidade Estadual

de Londrina.

Av. Robert Koch 60,

Londrina, $P R$

86038-350, Brasil.

anarigo@uel.br
}

\begin{abstract}
This research focused on opinions of mothers in Londrina whose infant children had died in 1997, attempting to demonstrate the feasibility and utility of studies on user satisfaction with health services. Opinions were analyzed according to type (positive, negative, or both positive and negative) and comments. The majority of mothers had positive opinions of the health services, especially prenatal care (75.6\%), while fewer (52.6\%) gave a positive assessment towards the hospital care provided to their infants who had died before they could be discharged from hospital. Most of the low opinions (21.1\%) concerned intrapartum care. Analysis of comments revealed satisfaction but also some problems in the relationship between the physician staff and patients; others related to the organization of services and medical techniques or guidance. This type of study can be useful for a more comprehensive evaluation of health care provision.
\end{abstract}

Key words Consumer Satisfaction; Child Health; Health Services

Resumo Este estudo teve como objetivos conhecer as opiniões de mães de crianças menores de um ano que morreram em 1997, em Londrina, sobre os serviços de saúde utilizados, e demonstrar a viabilidade e utilidade dos estudos de satisfação de usuários de serviços de saúde. As opiniões foram analisadas segundo o tipo (positiva, negativa ou mista) e quanto ao teor dos comentários. Para todos os serviços prevaleceram as opiniões positivas, com maior proporção para a atenção pré-natal (75,6\%), e menor para o atendimento hospitalar às crianças que não chegaram a ter alta do hospital após o nascimento (52,6\%). O atendimento ao parto recebeu a maior proporção de opiniões negativas (21,1\%). O teor dos comentários revelou satisfação e alguns problemas na relação profissional/equipe e usuários, na organização de serviços e com relação a técnicas e orientações. Conclui-se que estudos deste tipo fornecem elementos importantes para uma avaliação mais global da assistência à saúde.

Palavras-chave Satisfação dos Consumidores de Serviços de Saúde; Saúde Infantil; Serviços de Saúde 


\section{Introdução}

A avaliação dos serviços de saúde constitui uma importante ferramenta na busca pela qualidade da atenção, porém, no Brasil, na prática cotidiana, a avaliação não é realizada rotineiramente, muitas vezes por dificuldades metodológicas e operacionais (Silva \& Formigli, 1994). Além da escassez, os estudos sobre avaliação de serviços de saúde restringem-se principalmente a avaliações quantitativas, com poucos trabalhos sobre resolutividade e satisfação dos pacientes (Halal et al., 1994).

Entre os atributos que definem a qualidade do cuidado em saúde, citados por Donabedian (1990), um dos que se relaciona à satisfação do cliente é a aceitabilidade, definida como a adaptação do cuidado aos desejos, expectativas e valores do paciente e família. O mesmo autor acrescenta que a aceitabilidade depende de avaliação subjetiva, pelo paciente, sobre a efetividade e a eficiência do cuidado. Existem, porém, outros fatores relacionados à satisfação com o cuidado recebido, como a acessibilidade (capacidade de obter cuidado, quando necessário, de forma fácil e conveniente), a relação profissional-cliente e as comodidades do cuidado (conforto, higiene, alimentação, entre outros).

Segundo Vuori (1991), a medida da satisfação do paciente é um dos principais caminhos para se avaliar o resultado da atenção à saúde. Embora vários fatores possam afetar a percepção do usuário, destacam-se as experiências anteriores do cuidado recebido, em que condições elas se deram e o seu estado atual de saúde (Reis et al., 1990). Kloetzel et al. (1998:623) referem que "a satisfação expressa pelo usuário é um parâmetro facilmente mensurável, podendo tornar-se um instrumento útil na orientação de medidas corretivas".

No presente trabalho, objetivou-se verificar, entre mães de crianças que morreram antes de completar um ano de idade, sua opinião sobre os atendimentos recebidos nos serviços de pré-natal e de atendimento ao parto, bem como sobre os cuidados recebidos pela criança nos serviços ambulatoriais e hospitalares. Pretendeu-se também, demonstrar a viabilidade e utilidade dos estudos de satisfação de usuários de serviços de atenção à saúde. Destaca-se que este estudo faz parte de um trabalho mais amplo (Silva, 1999), que buscou, através de diversas fontes, caracterizar a mortalidade infantil e avaliar a assistência à saúde prestada desde a gestação até o momento do óbito da criança, no Município de Londrina, Estado do Paraná, em 1997.

\section{Metodologia}

A população de estudo constituiu-se de mães do total de crianças menores de um ano, residentes em Londrina, que morreram entre 1o de janeiro e 31 de dezembro de 1997. O município localiza-se ao norte do Estado do Paraná, contando com 421.343 habitantes em 1996 (IBGE, s.d.). A identificação das crianças foi realizada a partir das declarações de óbito, no Núcleo de Informações em Mortalidade (NIM), do Serviço Municipal de Saúde de Londrina, sendo todos os dados transcritos para formulário próprio da pesquisa.

Em 1997, ocorreram 107 óbitos de crianças menores de um ano residentes em Londrina, perfazendo um coeficiente de mortalidade infantil de 13,2 por mil nascidos vivos. Devido a partos gemelares, o número de mães observado foi de 103.

O instrumento utilizado para obtenção de dados e para registro da entrevista com a mãe, foi baseado no Estudo de Evento Sentinela: Morte Infantil, do Manual de Capacitação em Gerência de Unidades Básicas de Saúde (Santana et al., 1993), sendo composto de dados de identificação do óbito infantil, da entrevista com a mãe sobre características reprodutivas e condições sócio-econômicas familiares, além da utilização e opinião sobre serviços de saúde e, finalmente, de dados levantados em prontuários nos serviços de saúde.

Após esclarecimento dos objetivos da pesquisa e das garantias previstas na Resolução 196/96 do Conselho Nacional de Saúde (MS, 1997), as mães que concordaram em participar assinaram termo de consentimento. As entrevistas foram obtidas em sua própria residência, por uma das autoras (A. M. R. S.), obedecendo a um intervalo mínimo de sessenta dias após o óbito da criança, o qual foi estabelecido depois de algumas tentativas de entrevistas com intervalo menor, que não foram consentidas pelas mães, que alegaram não estarem dispostas a tocar no assunto, provavelmente devido ao trauma psicológico recente ocasionado pela perda da criança.

Dentre as 103 mães, foram realizadas 91 entrevistas $(88,3 \%)$. Dos 12 casos não entrevistados, o principal motivo foi a recusa da mãe em participar da pesquisa (oito casos); três já não residiam mais em Londrina quando o contato foi tentado e em apenas um caso o endereço não foi localizado. A maior parte das entrevistas foi realizada em até quatro meses após o óbito da criança $(74,7 \%)$ e apenas $5,5 \%$, após seis meses.

A análise dos dados foi subdividida nos diferentes momentos de atenção à saúde: ao pré- 
natal, ao parto, às crianças que nasceram em hospital e não chegaram a receber alta e às crianças que receberam alta e tiveram atendimento ambulatorial.

As opiniões das mães sobre os atendimentos foram categorizadas da seguinte forma: Opinião positiva - quando a opinião foi positiva e não houve relato de problemas no atendimento; Opinião negativa - quando a mãe manifestou somente opinião negativa sobre o serviço; Opinião positiva e negativa (mista) - quando a opinião foi positiva, mas com relato de problemas na assistência; Sem opinião - quando a mãe referiu que não tinha opinião formada a respeito do atendimento. Os comentários foram analisados quanto ao seu conteúdo e, para os atendimentos de pré-natal e parto, agrupados em "satisfação na relação médico ou equipe e paciente", "satisfação relacionada a questões técnicas e de orientações" e "satisfação com relação à organização do serviço”.

\section{Resultados}

\section{Opinião sobre o pré-natal}

Das 91 mães entrevistadas, 21 não fizeram prénatal e uma era adotiva, não opinando, portanto, sobre esse atendimento. Das 69 mães que realizaram pré-natal, 13 o fizeram em dois lugares e emitiram opinião sobre ambos, totalizando 82 opiniões, na maioria positivas (Tabela 1). Essas opiniões se referem, predominantemente, a atendimentos realizados no setor público $(81,7 \%)$, principalmente por unidades básicas de saúde da rede municipal.

Os comentários positivos relatados pelas mães revelaram, em sua maioria, satisfação na relação médico-paciente ou equipe-paciente. Afirmaram que foram bem tratadas e receberam atenção do médico e/ou equipe. Alguns depoimentos podem ilustrar essa percepção: "Fui super bem tratada, a mesma coisa de ser filha”, "eu estava nervosa, eu ia lá e elas [auxiliares de enfermagem] me davam conselhos", "bom, era bem tratada, o médico dava bastante atenção", “ótimo, médica atenciosa, não faltou em nenhum momento", "ótimo, o médico é um amigo", "tratam muito bem, são educados".

Ainda sobre os comentários positivos, os relacionados a questões mais técnicas e de orientações sobre o período da gravidez e parto foram os que apareceram em segundo lugar. Alguns exemplos são demonstrados a seguir: " $\mathrm{Ti}$ nha a pressão alta e fui bem monitorada", "faz ultra-som, faz tudo o que precisa", "lá tem palestra sobre parto, amamentação", "fui muito

\begin{tabular}{|c|c|c|}
\hline \multicolumn{3}{|c|}{$\begin{array}{l}\text { Distribuição das mães das crianças menores de um } \\
\text { ano que morreram em 1997, residentes em Londrina } \\
\text { segundo a opinião sobre o atendimento recebido no } \\
\text { pré-natal. }\end{array}$} \\
\hline Opinião & $\mathbf{n}$ & $\%$ \\
\hline Positiva & 62 & 75,6 \\
\hline Positiva e negativa & 8 & 9,8 \\
\hline Negativa & 10 & 12,2 \\
\hline Sem opinião & 2 & 2,4 \\
\hline Total & 82 & 100,0 \\
\hline
\end{tabular}

bem orientada”, "ensinaram o cuidado de um filho", "explicaram coisas que eu não sabia”.

Em menor proporção, apareceram os elogios referentes à organização do serviço: "não precisava levantar de madrugada para marcar consulta", "foi tudo fácil", "atendiam fora do dia quando eu precisava".

Quanto aos comentários negativos, prevaleceram os relacionados às questões técnicas e de orientação, como os descritos nos seguintes exemplos: "Eu acho a maior porcaria. Pra muitos que não têm nada é bom, mas pra mim, que tenho problemas, não foi. Fiz ultra-som por conta própria, deu pouco líquido, levei para o médico ver e ele mandou fazer repouso, mas eu fui para o hospital e me internaram até o parto", "o médico não me examinava", "não examina direito, todas as mulheres reclamam", "não gostei do pré-natal, não pelo jeito dele tratar, mas pelas condutas médicas. Foi descuido" (criança morreu de sífilis congênita e não houve tratamento na gestação), "o médico escondeu o problema da criança" (a criança nasceu com cardiopatia congênita, falecendo aos 2 meses), "não é muito bom não, eles não explicam, não conversam tudo o que deviam", "fiquei triste porque achava que se ela [a criança] tivesse algum problema eles teriam me falado", "faltou mais informação sobre a gravidez e o parto”, “aqui não presta, só mede a barriga, não fala nada".

Os problemas na organização do atendimento apareceram em segundo lugar, com destaque para reclamações quanto à demora no atendimento e dificuldade para agendar as consultas de pré-natal, conforme os exemplos: " a consulta era marcada às 15:00, e era atendida às 18:00 ou 19:00", "a minha mãe tinha de chegar às 6 horas da manhã para marcar consulta para mim".

As críticas a respeito do relacionamento interpessoal também foram relatadas: “Não gos- 
tei, os médicos são brutos, disseram que o meu nenê ia morrer mesmo" [mãe cujo filho nasceu com anencefalia, diagnosticada no pré-natal], "não recebi atenção do médico quando quis conversar sobre o parto", "eu falava e eles não escutavam".

\section{Opinião sobre o parto}

Das 91 mães entrevistadas, apenas uma não emitiu opinião sobre o atendimento ao parto, pois era adotiva. As opiniões mostraram-se bastante diferentes se comparadas às do pré-natal, com diminuição das opiniões positivas e aumento das negativas e mistas (Tabela 2). Apenas oito mães $(8,9 \%)$, tiveram seus partos em estabelecimentos privados e todas opinaram positivamente sobre o atendimento.

Os comentários positivos referidos pelas mães foram, na sua maioria, referentes ao relacionamento médico-paciente ou equipe-paciente, conforme os exemplos a seguir: "Muito bom, o jeito deles atender a gente, eles são ótimos, acalmam a mãe, não são estúpidos”, “ótimo, se preocupam com a gente, tentam acalmar por ser o primeiro, tentam passar força pra gente ter coragem; eles perguntam o nosso nome, mas nos chamam de mãezinha", "foi ótimo, porque no 1 o filho eu fui mal tratada, mas desta vez foram compreensivos, atenderam bem, foram prestativos".

Alguns depoimentos positivos demonstram que as opiniões também estão embasadas na questão técnica do atendimento, como por exemplo: "Toda hora era medida a pressão $e$ examinada pelo médico", "ótimo, não deixaram sofrer nada, fizeram o que puderam por mim", "Acho que foi feito tudo o que podiam, os médicos eram muito bons".

Os comentários positivos que envolvem as questões da organização do atendimento também foram mencionados, como se pode observar: "Foi bom, boa alimentação, higiene, foi ótimo o bebê ficar com a mãe", "lá atende bem, fui bem alimentada, tudo na hora certa", "fui bem atendida, toda hora estavam no quarto para ver como eu estava, o médico passava duas vezes", "foi um bom atendimento, rápido, sempre tinha uma enfermeira comigo, o médico toda hora estava lá".

Entre os comentários negativos, houve predominância de críticas às questões técnicas, que podem ser observadas nos seguintes depoimentos: "Foi mal né, só foi feito toque quando eu cheguei, nasceu na maca, debaixo de um ventilador", "acho que ter esperado 12 horas para fazer cesárea... agravou as condições da criança”, “...tive infecção nos pontos...", "nasceu sem recurso, no pronto-socorro, faltou mais cuidado, recebi alta no mesmo dia do parto", "mesmo que o médico seja bom, as condutas são para o SUS".

Os comentários negativos envolvendo o relacionamento interpessoal também foram freqüentes, com depoimentos como os exemplos a seguir: "São muito brutos, o médico gritava comigo, fui mal tratada pela enfermeira, dizendo que a criança tinha má formação por eu ter tentado aborto", "não gostei, pela falta de interesse da equipe. Eu queria fazer laqueadura e a médica foi estúpida e disse que eu era muito jovem e o bebê era excepcional", "Nota zero, não davam atenção, pois estavam numa festa de final de ano".

Críticas que envolveram a parte da organização do serviço também foram observadas, conforme alguns relatos: “...o médico que fez o parto não foi o mesmo que estava acompanhando a internação...", "não tem campainha no quarto e se gritarmos acham ruim...", “...demorei para conseguir entrar no pronto-socorro...”, “...demorou para vir o médico...”.

\section{Opinião sobre o atendimento}

hospitalar à criança que não recebeu

alta após o nascimento

Entre as 107 crianças que morreram, 65 não receberam alta após o nascimento. Devido a gestações gemelares, o número de mães observado foi 61 e destas, quatro não foram entrevistadas, restando, portanto, 57 .

Mais da metade das mães emitiu opinião positiva sobre o atendimento hospitalar à criança que não recebeu alta, porém destaca-se uma grande parcela que afirmou não ter opinião (Tabela 3), pois, na maior parte desses casos, não puderam presenciar o atendimento prestado a seus filhos, devido à precocidade do óbito.

Os comentários positivos relatados, na maioria das vezes, expressavam confiança devido à tecnologia de unidades de terapia intensiva (UTI), ao cuidado dos profissionais, bem como ao esclarecimento sobre o estado que a criança apresentava, como se pode verificar: “...ótimo, sempre estavam em cima da criança e falavam como ela estava...", "o atendimento foi muito bom, tinha quatro pediatras cuidando dela, fo $i$ feito tudo o que podia...”, “... gostei do atendimento e foram honestos...”, “... se preocuparam bastante com ela, o que o homem podia fazer foi feito...", “ótimo, fazem tudo o que é possível na UTI, com todos os aparelhos, só dependia da criança...", "foi bem atendida, foi para a UTI".

Neste estudo, entre os comentários negativos, pôde-se observar que algumas mães não 
acreditaram nas informações recebidas sobre o tratamento que seus filhos receberam, como por exemplo: “...não acredito que eles operaram o nenê, não confiei muito bem...”, “...tenho desconfiança se as crianças receberam os medicamentos caros para o pulmão, por ser do SUS...".

Outras mães relacionam o óbito a problemas ocorridos no trabalho de parto ou no parto, propriamente dito, como os exemplos abaixo: "Acho que forçaram o parto e o intestino rompeu, pois no ultra-som estava normal, acho que foi no hospital”, “...se tivesse sido cesárea, ela não tinha morrido...”, “...o parto devia ter sido mais rápido, pois o oxigênio que faltou forçou o pulmão...”.

Houve comentários negativos a respeito da equipe de saúde, relacionados à questão técnica e outros, e à falta de informações claras sobre o estado da criança. Os depoimentos a seguir ilustram essas afirmações: "Ela nasceu à noite e quando eu perguntava sobre ela, diziam que estava bem, mas de manhã, quando eu vi, achei que estava praticamente morta, estava largada. Não achei bom, pois se eles tivessem recurso, ela não tinha morrido. E devia ter, pois uma maternidade é própria para isso”, “...tive muitas dúvidas, falta de esclarecimentos, pois nem sabia que a criança estava com pneumonia, o pediatra dela faltou muito, só via a criança no dia que estava de plantão...”, “...todas as vezes que eu fui até a UTI, as enfermeiras estavam cuidando das outras crianças...”, “...acho que foi mal atendida, informavam que a criança estava bem e não estava. Não confio no atendimento de enfermagem, porque largam as crianças...”, “...a lentidão dos exames e o adiamento da cirurgia foram fatais para o meu filho...".

\section{Opinião sobre o atendimento ambulatorial}

Entre as 42 crianças que receberam alta hospitalar após o nascimento, seis não receberam acompanhamento ambulatorial e oito mães não foram entrevistadas, ficando, então, 28 opiniões. A maioria das mães teve, também, opinião positiva sobre o atendimento ambulatorial da criança (Tabela 4). Entre as mães entrevistadas, apenas uma havia utilizado o serviço privado (convênio) para o acompanhamento de seu filho e a opinião foi negativa.

A maior parte das opiniões exclusivamente positivas não tiveram justificativas para a satisfação, visto que eram simplesmente: "muito bom, sem reclamação" ou ainda, "atenderam bem”. No entanto, algumas fizeram comentários diversos, como os exemplificados a seguir: “Atenderam bem, o pediatra era bom e as enfer-
Tabela 2

Distribuição das mães das crianças menores de um ano que foram a óbito em 1997, residentes em Londrina, segundo a opinião sobre o atendimento recebido no parto.

\begin{tabular}{lrr}
\hline Opinião & $\mathbf{n}$ & $\%$ \\
\hline Positiva & 55 & 61,1 \\
Positiva e negativa & 16 & 17,8 \\
Negativa & 19 & 21,1 \\
Total & 90 & 100,0 \\
\hline
\end{tabular}

Tabela 3

Distribuição das mães das crianças menores de um ano que foram a óbito em 1997 e que não receberam alta após o nascimento, residentes em Londrina, segundo a opinião sobre o atendimento durante a internação da criança.

\begin{tabular}{lrr}
\hline Opinião & $\mathbf{n}$ & \multicolumn{1}{c}{$\%$} \\
\hline Positiva & 30 & 52,6 \\
Positiva e negativa & 6 & 10,5 \\
Negativa & 11 & 19,3 \\
Sem opinião & 10 & 17,6 \\
Total & 57 & 100,0 \\
\hline
\end{tabular}

Tabela 4

Distribuição das mães das crianças menores de um ano que foram a óbito em 1997, residentes em Londrina, segundo a opinião sobre o atendimento ambulatorial à criança.

\begin{tabular}{lrr}
\hline Opinião & $\mathbf{n}$ & $\%$ \\
\hline Positiva & 17 & 60,7 \\
Positiva e negativa & 5 & 17,9 \\
Negativa & 4 & 14,3 \\
Sem opinião & 2 & 7,1 \\
Total & 28 & 100,0 \\
\hline
\end{tabular}


meiras também”, “fui bem atendida, mandavam tirar a roupa para examinar", "muito bom, atendiam certinho, com paciência, carinho $e$ educação".

Os comentários negativos, algumas vezes, estavam relacionados à doença que causou a morte da criança, como por exemplo: "Foi falha, não recebi informações corretas sobre as vacinas" (criança morreu devido a meningite por Haemophilus tipo B, antes da vacina ser disponibilizada nas unidades públicas), "na verdade, eu reclamo muito dali, porque eu levava duas vezes por dia e as enfermeiras me tratavam mal, ninguém observou o crescimento da cabeça da criança" (criança morreu durante cirurgia para retirada de um tumor na cabeça).

Outras opiniões mistas (positivas e negativas): "O médico não é grande coisa, mas as enfermeiras explicam tudo direitinho", "a médica é muito dedicada e carinhosa, mas não entende de doença”, "foi bem atendido, só não entendo como não perceberam antes que ele tinha problema de coração".

\section{Discussão}

Um aspecto a se destacar, no presente trabalho, foi a considerável proporção de informações obtidas, comparando com estudo realizado no distrito de Pau da Lima, em Salvador, cuja perda atingiu 76,6\% nas entrevistas domiciliares realizadas cerca de dois anos após o óbito da criança (Formigli et al., 1996). Além de diferenças nas populações pesquisadas, é provável que o tempo decorrido entre o óbito da criança e a entrevista com a mãe tenha sido fator determinante para reduzir essa perda em Londrina, onde a maioria das entrevistas se realizou em até quatro meses após o óbito da criança, diminuindo a possibilidade de as famílias mudarem de residência. Este achado corrobora a afirmação de que sistemas de informação de mortalidade infantil ágeis, descentralizados e desburocratizados são imprescindíveis neste tipo de avaliação (Formigli et al., 1996).

Não obstante o estudo tenha enfocado usuárias que experimentaram um desfecho negativo (a morte de seus filhos), as opiniões positivas prevaleceram nos quatro níveis de atenção pesquisados. Este tipo de resultado é considerado como uma dificuldade por Carr-Hill (1992), pois ao administrador interessa mais conhecer o que está errado, enquanto que as respostas dos usuários apontam mais o que está certo.

Outra dificuldade é o receio dos usuários em manifestar opiniões negativas, principalmente quando o entrevistador faz parte da equipe que presta assistência. Oliveira (1985), em estudo sobre a opinião acerca do atendimento de um posto de saúde, também em Londrina, destacou que a população mostrou-se receosa e com dificuldades para responder (ausência de resposta variou entre 35,0 e $45,0 \%$ ). No presente estudo, possivelmente, essa dificuldade foi contornada, pois a entrevistadora não fazia parte do quadro de recursos humanos de instituição prestadora de serviços de saúde, além de ter sido garantido total anonimato às entrevistadas.

Outro obstáculo nesse tipo de avaliação é a ausência de um padrão de referência de qualidade, conhecido pelos usuários, para avaliação da assistência. Sucupira (1981, apud Oliveira, 1985) refere que a população necessita de contato com diferentes serviços, para auxiliar na formação deste padrão.

Problema adicional, levantado por Thiollent (1982), é que algumas vezes a forma de abordagem dos entrevistados pode induzir a um tipo de resposta (o sim, por exemplo, tende a ser favorecido). Nesta investigação, a entrevista foi baseada em roteiro com perguntas abertas, do tipo "qual a sua opinião sobre o atendimento pré-natal?" ou "você teve problemas no atendimento?", o que pode ter amenizado a indução das respostas.

Observou-se ainda que, apesar do óbito da criança, a proporção de opiniões positivas sobre o pré-natal $(75,6 \%)$ foi um pouco superior à observada por Gomes (1995), entre 110 puérperas no Rio de Janeiro $(67,4 \%)$. Este achado pode refletir o fato de que grande parte das mães entrevistadas não relaciona o atendimento à gestação ao óbito da criança. Como uma parcela dos óbitos ocorreu por motivos diversos aos relacionados à gestação, este fato é compreensível. Na Austrália, porém, Laslett et al. (1997) observaram proporção ainda maior de opiniões positivas $(90,1 \%)$, sobre o atendimento pré-natal em diferentes serviços.

O teor dos comentários sobre a atenção prénatal coincidem com o trabalho de Nogueira (1994), em que as opiniões positivas das gestantes ouvidas, muitas vezes estavam relacionadas à atenção dos médicos às pacientes, sendo este um importante aspecto da qualidade dos serviços de saúde, na percepção dessas usuárias.

Os comentários sobre questões técnicas e de orientação, que predominaram entre as opiniões negativas em relação ao pré-natal, podem estar indicando que mães que passaram por um evento negativo, como a morte de seu filho, tenham se motivado a obter informações adicionais sobre o processo que ocasionou o óbito, 
levando-as a questionar a qualidade da atenção à saúde em condutas específicas (não tratamento de sífilis, não indicação de vacina, falta de informação sobre anomalia da criança, etc.). A falta de orientação e de comunicação durante o pré-natal foi percebida, assim, como importante problema para muitas das mulheres entrevistadas, estando estreitamente relacionada ao aspecto de relacionamento interpessoal. De forma semelhante, no Rio de Janeiro, grande parte das puérperas relatou não ter recebido informações sobre preparação para o parto $(79,8 \%)$ ou sobre aleitamento materno (77,5\%) (Gomes, 1995).

Quanto ao parto, proporção bem maior de opiniões positivas $(90,0 \%)$, foi observada no Rio de Janeiro (Gomes, 1995), fato considerado plausível, dado que grande parte dos óbitos, no presente trabalho, ocorreu logo após o parto, no período neonatal precoce, o que pode ter influenciado negativamente a opinião das mães sobre esse tipo de atendimento.

Os depoimentos das mães em relação ao parto, reforçam a afirmação de que o ambiente físico, a postura adotada pela equipe, as emoções e sentimentos das pessoas que circundam a cena de parto são considerados como fatores determinantes do processo, podendo contribuir ou não para um parto e nascimento harmoniosos (Carvalho, 1993). Atualmente, quando a grande maioria dos partos é realizada em hospitais, há que se considerar não somente os aspectos biológicos do atendimento, mas também os psicoafetivos, culturais e sociais, relacionados à maternidade. Entre as principais atividades que completam a atenção médica, destacam-se a educação e a preparação física e psíquica da mãe para o parto e amamentação, a participação do pai e familiares durante o parto e o contato precoce dos pais com seus filhos (Schwarcz et al., 1995). Para um atendimento adequado, faz-se necessário contemplar, na formação ou educação da equipe, conhecimentos sobre aspectos sociais, culturais, antropológicos e éticos do nascimento e habilidades de comunicação que favoreçam o relacionamento profissional-cliente durante esse período (Proctor, 1998; Romito \& Zalateo, 1992).

A respeito do atendimento hospitalar às crianças, muitas mães referiram não poder opinar em razão da precocidade do óbito. Entre os comentários negativos, ressaltam-se os que denotam desconfiança com a qualidade do atendimento prestado e a falta de esclarecimentos da situação da criança pela equipe de saúde. Já entre os positivos, percebe-se, em diversos depoimentos, a valorização da tecnologia de unidades de terapias intensivas no atendimento ao recém-nascido. Em trabalho realizado na Espanha (Vidal et al., 1992), embora com uma taxa de resposta de apenas $27,2 \%$ aos questionários enviados, houve $95,0 \%$ de opiniões positivas relacionadas ao cuidado médico e 93,0\% de satisfação com as informações recebidas sobre a situação de crianças que morreram em UTI.

Com relação ao atendimento ambulatorial de crianças que morreram após a alta hospitalar, o resultado de apenas $60,7 \%$ de opiniões positivas, neste trabalho, é bastante inferior ao encontrado por Franco \& Campos (1998), em Campinas (São Paulo), em que 98,2\% dos pais estavam satisfeitos com esse tipo de atendimento. Essa diferença pode ser explicada pelo desfecho negativo no presente estudo, tendo, inclusive, relatos de problemas no atendimento relacionado à própria doença que levou à morte.

O presente trabalho buscou avaliar, utilizando metodologia quantitativa e qualitativa, a percepção de mães com relação à qualidade da atenção à saúde prestada durante todo o processo que resultou em óbito infantil, incluindo a fase de gestação. Partiu-se de um desfecho negativo (o óbito infantil) para avaliar o processo da atenção à saúde e detectar possíveis falhas nesse processo. As entrevistas com as mães possibilitaram a obtenção de diversas informações que não seriam captadas por outros meios. Aliadas a consultas a prontuários, constituem-se em ferramenta imprescindível para uma avaliação mais global da assistência prestada e, conseqüentemente, para a definição de propostas mais eficazes de intervenção, levando-se em consideração a perspectiva e desejos dos usuários. Vale ressaltar que, com base na análise de trabalhos anteriores, optou-se por um intervalo relativamente curto após o óbito da criança, para a realização da entrevista. Esse fato que parece ter sido o grande responsável pela perda relativamente pequena de informações, sendo esta estratégia recomendada para estudos semelhantes.

Em conclusão, acredita-se que este tipo de estudo é importante de ser realizado, sendo factível e de custo relativamente baixo, podendo contribuir com os gestores do sistema e profissionais de saúde para a detecção de aspectos passíveis de melhoria na assistência, com vistas à organização de serviços mais acessíveis, humanizados e com impacto positivo no nível de saúde. 


\section{Referências}

CARR-HILL, R. A., 1992. The measurement of patient satisfaction. Journal of Public Health Medicine, 14:236-249.

CARVALHO, M. L., 1993. Mortalidade Neonatal e Aspectos da Qualidade da Atenção à Saúde na Região Metropolitana do Rio de Janeiro em 1986/ 1987. Dissertação de Mestrado, Rio de Janeiro: Escola Nacional de Saúde Pública, Fundação Oswaldo Cruz.

DONABEDIAN, A., 1990. The seven pillars of quality. Archives of Pathology \& Laboratory Medicine, 14: 1115-1118.

FORMIGLI, V. L. A.; SILVA, L. M. V.; CERDEIRA, A. J. P.; PINTO, C. M. F.; OLIVEIRA, R. S. A.; CALDAS, A. C.; VILAS-BOAS, M. J. B.; FONSECA, A. C.; SOUZA, L. S. F.; SILVA, L. R. \& PAES, M. S. F., 1996. Avaliação da atenção à saúde através da investigação de óbitos infantis. Cadernos de Saúde Pública, 12(Sup. 2):33-41.

FRANCO, S. C. \& CAMPOS, G. W. S., 1998. Avaliação da qualidade de atendimento ambulatorial em um Hospital Universitário. Cadernos de Saúde Pública, 14:61-70.

GOMES, M. A. S. M., 1995. Aspectos da Qualidade do Atendimento à Gestação e ao Parto através da Percepção das Usuárias. Dissertação de Mestrado, Rio de Janeiro: Instituto Fernandes Figueira, Fundação Oswaldo Cruz.

HALAL, I. S.; SPARRENBERGER, F.; BERTONI, A. M.; CIACOMET, C.; SEIBEL, C. E.; LAHUDE, F. M.; MAGALHÃES, G. A.; BARRETO, L. \& LIRA, R. C. A., 1994. Avaliação da qualidade de assistência primária à saúde em localidade urbana da Região Sul do Brasil. Revista de Saúde Pública, 28:131136.

IBGE (Fundação Instituto Brasileiro de Geografia e Estatística), s.d. Contagem da População 1996: Dados Distritais - População por Situação e Sexo. Curitiba: Departamento Regional Sul, Divisão de Pesquisa do Paraná, IBGE. (mimeo.)

KLOETZEL, K.; BERTONI, A. M.; IRAZOQUI, M. C.; CAMPOS, V. P. G. \& SANTOS, R. N., 1998. Controle de qualidade em atenção primária à saúde. I - A satisfação do usuário. Cadernos de Saúde Pública, 14:623-628.

LASLETT, A. M.; BROWN, S. \& LUMLEY, J., 1997. Women's views of different models of antenatal care in Victoria, Australia. Birth, 24:81-89.

MS (Ministério da Saúde), 1997. Diretrizes e Normas Regulamentadoras de Pesquisa Envolvendo Seres Humanos. Brasília: Programa Nacional de Doenças Sexualmente Transmissíveis, Conselho Nacional de Saúde, Secretaria de Assistência à Saúde, MS.
NOGUEIRA, M. I., 1994. Assistência Pré-Natal: Prática de Saúde a Serviço da Vida. São Paulo: Editora Hucitec.

OLIVEIRA, M. S. M., 1985. Utilização e Opinião da População sobre um Posto de Saúde Periférico em Londrina, Paraná. Dissertação de Mestrado, São Paulo: Faculdade de Saúde Pública, Universidade de São Paulo.

PROCTOR, S., 1998. What determines quality in maternity care? Comparing the perceptions of childbearing women and midwives. Birth, 24:81-89.

REIS, E. J. F. B.; SANTOS, F. P.; CAMPOS, F. E.; ACÚRCIO, F. A.; LEITE, M. T. T.; LEITE, M. L. C.; CHERCHIGLIA, M. L. \& SANTOS, M. A, 1990. Avaliação da qualidade dos serviços de saúde: Notas bibliográficas. Cadernos de Saúde Pública, 6:50-61.

ROMITO, P. \& ZALATEO, C., 1992. Social history of a research project: A study on early post-partum discharge. Social Science and Medicine, 34:227235.

SANTANA, J. P.; GALVÃO, E. A.; SANTOS, I.; MANDELLI, M. J.; FEKETE, M. C.; PENNA, M. L. F. \& MOTA, M.V., 1993. Capacitação em Gerência de Unidades Básicas de Saúde do Distrito Sanitário. Série Desenvolvimento de Recursos Humanos. Brasília: Organização Pan-Americana da Saúde.

SCHWARCZ, R.; DIAZ, A. G.; FESCINA, R.; DE MUCIO, B.; BELITZKI, R. \& DELGADO, L., 1995. Salud Reproductiva Materna Perinatal. Atención Prenatal y del Parto de Bajo Riesgo. Montevideo: Centro Latinoamericano de Perinatología y Desarrollo Humano Organizacíon Panamericana de la Salud/Organización Mundial de la Salud.

SILVA, A. M. R., 1999. A Mortalidade Infantil e a Assistência à Saúde - Londrina, 1997. Dissertação de Mestrado, Londrina: Centro de Ciências da Saúde, Universidade Estadual de Londrina.

SILVA, L. M. V. \& FORMIGLI, V. L. A., 1994. Avaliação em saúde: Limites e perspectivas. Cadernos de Saúde Pública, 10:80-91.

THIOLLENT, M. J. M., 1982. Crítica Metodológica, Investigação Social e Enquete Operária. 3a Ed. São Paulo: Editora Polis.

VIDAL, J. K.; VAZQUEZ, M. S.; FANES, M. I.; PALAU, R. F. \& HERNANDO, J. M., 1992. Atención a los padres de recién nacidos fallecidos en nuestra unidad neonatal. Anales Españoles de Pediatría, 36: 419-422.

VUORI, H. A., 1991. Qualidade da saúde. Divulgação em Saúde para Debate, 3:17-25.

Recebido em 28 de dezembro de 2000

Versão final reapresentada em 6 de dezembro de 2001 Aprovado em 6 de março de 2002 